\title{
Chow motives of abelian type over a base
}

\section{Vladimir Guletskiı ${ }^{1}$}

Received: 2 February 2018 / Accepted: 19 June 2018 / Published online: 20 August 2018

(c) The Author(s) 2018

\begin{abstract}
Let $S$ be a Noetherian scheme, let $X$ be a smooth projective scheme over $S$, whose fibres are connected curves of genus $g$, and let $J$ be the Jacobian scheme of the relative curve $X$ over $S$. We generalise the theorem due to Rolph Schwarzenberger and prove that if $S$ is integral and normal, and the structural morphism admits a section, then there exists a locally free sheaf $\mathcal{F}_{d}$ on $J$, such that the relative symmetric power $\operatorname{Sym}^{d}(X / S)$ is isomorphic to the projective bundle $\mathbb{P}\left(\mathcal{F}_{d}\right)$ over $J$, provided $d>2 g-2$, and the ample divisor is $\operatorname{Sym}^{d-1}(X / S)$, embedded into $\operatorname{Sym}^{d}(X / S)$ by the section of the structural morphism from $X$ to $S$. Then we use this result to generalise the theorem due to Shun-Ichi Kimura: if $S$ is an integral regular scheme, separated and of finite type over a Dedekind domain, then all relative Chow motives of abelian type over $S$ are finite-dimensional.
\end{abstract}

Keywords Relative curves - Jacobian scheme $\cdot$ Picard bundles $\cdot$ Symmetric powers · Riemann-Roch theorem - Chow motives · Chern class · Projective bundle theorem . Traces $\cdot$ Numerical equivalence $\cdot$ Nilpotent correspondences

Mathematics Subject Classification $14 \mathrm{C} 15 \cdot 14 \mathrm{C} 25 \cdot 14 \mathrm{G} 40$

\section{Introduction}

A well-known theorem due to Kimura asserts that the motive of a smooth projective curve over a field is finite-dimensional, see [11, Theorem 4.2]. Though not stressed explicitly, an important role in its proof is played by a much older result saying that the $n$-th symmetric power of a smooth projective curve is a projective bundle over its Jacobian, for a sufficiently big $n$, and that the $(n-1)$-th symmetric power, being embedded into the $n$-th power by a fixed point, is the corresponding relative ample divisor on this bundle. Most likely, this result was already known to Chow [4], and

Vladimir Guletskiı̌

vladimir.guletskii@liverpool.ac.uk

1 Department of Mathematical Sciences, University of Liverpool, Peach Street, Liverpool L69 7ZL, England, UK 
possibly to Weil [21]. The modern presentation in terms of Picard sheaves was given by Schwarzenberger in the early sixties, see [17, Proposition 11], based on Mattuck's work $[13,14]$, and obviously under the influence of Grothendieck's "Technique de descente et théorèmes d'existence en géométrie algébrique" and EGA.

The aim of this note is to examine to which extent one can generalise both results working over a general Noetherian base scheme. We think that the most relative version of Schwarzenberger's Proposition 11 is this.

Theorem A Let $S$ be an arbitrary Noetherian integral and normal base scheme, let $X$ be a smooth projective scheme of relative dimension 1 over $S$ whose fibres are connected curves of genus $g$, and let $J$ be the Jacobian scheme of the relative curve $X$ over $S$. Assume also that the structural morphism from $X$ to $S$ admits a section. Then, for any integer $d>2 g-2$, there exists a locally free sheaf $\mathcal{F}_{d}$ on the Jacobian scheme $J$, such that the d-th symmetric power $\operatorname{Sym}^{d}(X / S)$ is isomorphic to the projective bundle $\mathbb{P}\left(\mathcal{F}_{d}\right)$ over J. Moreover, the $(d-1)$-th symmetric power $\operatorname{Sym}^{d-1}(X / S)$, being embedded into $\operatorname{Sym}^{d}(X / S)$ by the section of the structural morphism, is the corresponding relative ample divisor on that bundle.

To generalise Kimura's theorem, the only restrictions on the base scheme $S$ come from the elements of intersection theory needed to construct an appropriate category of Chow motives over $S$.

Theorem B Let $S$ be an integral regular scheme of finite type over a Dedekind domain ${ }^{1}$, let $\mathrm{M}(S)$ be the category of Chow motives over $S$ with coefficients in $\mathbb{Q}$, and let $\mathrm{A}(S)$ be the full pseudo-abelian tensor subcategory generated additively and tensorially by motives of relative curves in $\mathrm{M}(S)$. Then all motives in $\mathrm{A}(S)$ are finite-dimensional.

Similarly to the absolute case, this theorem implies the following nilpotency result, which can be deduced applying [2, Proposition 9.1.14].

Corollary C Let $\eta$ be the generic point of $S$, let $L$ be a field extension of the residue field $\kappa(\eta)$, and let $\xi=\operatorname{Spec}(L)$ be the spectrum of the field L. Any endomorphism of a motive in $\mathrm{A}(S)$, whose pullback to $\xi$ is nilpotent modulo numerical equivalence, is nilpotent modulo rational equivalence over the base scheme $S$.

As a toy model, let $\varepsilon_{5}$ be a 5-th primitive root of the unity in $\mathbb{C}$, let $\mathbb{Q}\left(\varepsilon_{5}\right)$ be the minimal subfield containing $\varepsilon_{5}$ in $\mathbb{C}$, and let $\mathbb{Z}\left[\varepsilon_{5}\right]$ be the ring of integers in the number field $\mathbb{Q}\left(\varepsilon_{5}\right)$. Let, furthermore, $F$ be the Fermat scheme given by the form $x_{0}^{5}+x_{1}^{5}+x_{2}^{5}+x_{3}^{5}$ in $\mathbb{P}_{\mathbb{Z}\left[\varepsilon_{5}\right]}^{3}$, and let $X$ be the quotient by the standard action of the cyclic group $\mu_{5}$ on the scheme $F$. Using Theorem B and Corollary C, one can show that the relative Chow motive of the Fermat scheme $F$, considered outside points of bad reduction, is finite-dimensional, and therefore so is the relative motive of the Godeaux scheme $X$. Applying the same nilpotency result again, one shows that any two sections of the structural morphism from $X$ to $\operatorname{Spec}\left(\mathbb{Z}\left[\varepsilon_{5}\right]\right)$ are rationally equivalent as 1 -cycles on the 3-dimensional scheme $X$, outside a finite number of primes in $\mathbb{Z}$ [ $\left.\varepsilon_{5}\right]$.

The same applies to any 3-dimensional scheme over a Dedekind domain, if we know that its generic fibre is a smooth projective surface with trivial second transcendental cohomology group, whose Chow motive is finite-dimensional.

1 including a field 


\section{The proof of Theorem A}

Let $S$ be an arbitrary Noetherian scheme, and let $f: X \rightarrow S$ be a scheme smooth and projective over $S$, whose fibres at points $s \in S$ are smooth projective integral curves over the residue fields $\kappa(s)$. Under these assumptions, the structural morphism $f$ is flat and proper, and its fibres are reduced and connected. Therefore, we have an isomorphism $\mathcal{O}_{S} \stackrel{\sim}{\longrightarrow} f_{*} \mathcal{O}_{X}$ which holds universally, see [7, bottom of p. 260]. Let $\mathcal{P i c}_{X / S}$ be the Picard functor sending any locally Noetherian scheme $T$ over $S$ to the group $\operatorname{Pic}\left(X_{T}\right) / \operatorname{pr}_{T}^{*} \operatorname{Pic}(T)$, where $X_{T}=X \times{ }_{S} T$, for each scheme $Y$ the symbol $\operatorname{Pic}(Y)$ denotes the group of classes of line bundles on $Y$, and $\operatorname{pr}_{T}: X_{T} \rightarrow T$ is the projection. Assume also that the structural morphism $f$ from $X$ to $S$ has a section $\sigma: S \rightarrow X$. Notice that since the isomorphism from $\mathcal{O}_{S}$ to $f_{*} \mathcal{O}_{X}$ holds universally and the morphism $f$ has a section, the presheaf $\mathcal{P i c}_{X / S}$ is a sheaf in Zariski, étale and fppf topologies by Theorem 9.2.5 in Kleiman's paper [7], and therefore there is no ambiguity in choosing the Picard functor.

Let Pic $(X / S)$ be the Picard scheme of $X / S$ representing the functor $\mathcal{P i c}_{X / S}$, which exists either by Theorem 9.4.8 or by Theorem 9.4.18.1 in loc. cit. Let also $\operatorname{Pic}^{d}(X / S)$ be the open and closed subscheme of $\operatorname{Pic}(X / S)$, representing the Picard subfunctor $\operatorname{Pic}_{X / S}^{d}$ of line bundles of degree $d$ over the base scheme $S$, see [3, Theorem 1, p. 252].

The effective counterpart of the $d$-th Picard functor is provided by the functor $\mathcal{D i v}_{X / S}^{d}$ sending $T$ to the set of effective relative Cartier divisors of degree $d$ on $X_{T} / T$, see Section 9.3 in Kleiman's paper [7]. The functor $\mathcal{D i v}_{X / S}^{d}$ is representable by the $d$-th symmetric product $\operatorname{Sym}^{d}(X / S)$ of $X$ over $S$. The latter is the quotient of the $d$-fold fibered product of $X$ over $S$ by the natural action of the symmetric group of permutations of $n$ elements.

Notice that this quotient is a scheme over $S$, and it exists because any projective morphism is an AF-morphism, i.e., for any point $s \in S$ and for any finite collection $\left\{x_{1}, \ldots, x_{l}\right\}$ of points in the fibre $X_{s}$ there exists a Zariski open subset $U$ in $X$, such that $\left\{x_{1}, \ldots, x_{l}\right\} \subset U$, and the composition $U \rightarrow X \rightarrow S$ is a quasi-affine morphism of schemes, see [16, Exposé V]. Moreover, the structural morphism from $\operatorname{Sym}^{d}(X / S)$ to $S$ is normal, in the sense of [18, Tag 0390]. Notice that the $d$-th symmetric power $\operatorname{Sym}^{d}(X / S)$ is isomorphic, over $S$, to the Hilbert scheme $\operatorname{Hilb}^{d}(X / S)$ representing the Hilbert functor $\mathcal{H} i b_{X / S}^{d}$, see Proposition 6.3.9 in Deligne's "Cohomologie à supports propres" in [20].

The canonical morphism of functors

$$
\operatorname{Div}_{X / S}^{d} \rightarrow \operatorname{Pic}_{X / S}^{d}
$$

sending any relative Cartier divisor $D$ of degree $d$ on $X{ }_{S} T$ to the invertible sheaf $\mathcal{O}_{X}(D)$, induces the corresponding morphism of schemes

$$
\operatorname{Sym}^{d}(X / S) \rightarrow \operatorname{Pic}^{d}(X / S) .
$$

Let $Z$ be the image of the section $\sigma$, considered with the induced reduced scheme structure on it. Then $Z$ is a relative Cartier divisor of degree 1 on $X$ over $S$, see [3, 
p. 254] or [20, p. 437] (or, alternatively, use [18, Tag 0B9D]). For any scheme $T$ of finite type over $S$, the section $\sigma: S \rightarrow X$ of the structural morphism $f: X \rightarrow S$ induces a section $\sigma_{T}: T \rightarrow X \times{ }_{S} T$ of the projection $\mathrm{pr}_{T}$, whose image $Z_{T}$ is a relative Cartier divisor of degree 1 on $X_{T}$ over $T$. The corresponding line bundle $\mathcal{O}_{X_{T}}\left(Z_{T}\right)$ is of degree 1 on $X \times{ }_{S} T$. Then, for any line bundle on $X_{T}$ of degree $d$, the line bundle

$$
\mathcal{L} \otimes \mathcal{O}_{X_{T}}\left(Z_{T}\right)^{-d}
$$

is of degree 0 , and we obtain a morphism of functors

$$
\operatorname{Pic}_{X / S}^{d} \rightarrow \mathcal{P i c}_{X / S}^{0}
$$

sending a class represented by a line bundle $\mathcal{L}$ to the class represented by the line bundle $\mathcal{L} \otimes \mathcal{O}_{X_{T}}\left(Z_{T}\right)^{\otimes-d}$ on $X \times{ }_{S} T$. It indices a morphism of schemes

$$
\operatorname{Pic}_{X / S}^{d} \rightarrow J
$$

where

$$
J=\operatorname{Pic}^{0}(X / S)
$$

is the Jacobian scheme of the relative curve $X$ over $S$. Composing the latter with the above morphism $\operatorname{Sym}^{d}(X / S) \rightarrow \operatorname{Pic}^{d}(X / S)$ we obtain the morphism

$$
s_{d}: \operatorname{Sym}^{d}(X / S) \rightarrow J
$$

Let $P$ be the Poincare divisor on $X \times J$, i.e., a relative Cartier divisor such that the corresponding line bundle $\mathcal{P}=\mathcal{O}_{X \times J}(P)$ is a universal line bundle of degree 0 on $X \times{ }_{S} J$. It is convenient to normalise the Poincaré bundle $\mathcal{P}$ getting

$$
\left(\operatorname{id}_{X} \times{ }_{S} 0\right)^{*} \mathcal{P} \simeq \mathcal{O}_{X} \quad \text { and } \quad\left(g \times \operatorname{id}_{J}\right)^{*} \mathcal{P}=\mathcal{O}_{J},
$$

where $0: S \rightarrow J$ is the zero-section of the structural morphism from $J$ to $S$. Let

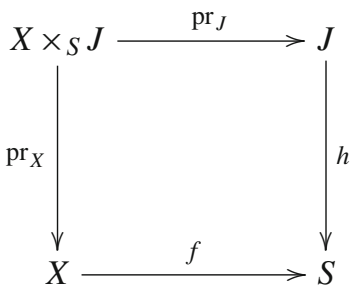

be the two obvious projections. For each positive integer $d$ let

$$
\mathcal{G}_{d}=\mathcal{P} \otimes \mathrm{pr}_{X}^{*} \mathcal{O}_{X}(Z)^{\otimes d}=\mathcal{P} \otimes \mathcal{O}_{X_{J}}\left(Z_{J}\right)^{\otimes d} .
$$

The direct image 


$$
\mathcal{E}_{d}=\operatorname{pr}_{J *} \mathcal{G}_{d}
$$

is a coherent sheaf on $J$ by [9, Chapter III, Theorem 8.8]. Generalising the definition in $\left[17\right.$, p. 258], one says that $\mathcal{E}_{d}$ is a relative Picard sheaf on the Jacobian scheme $J$.

Let $g$ be the genus of the fibres of the structural morphism $f: X \rightarrow S$.

Lemma $2.1 \mathcal{E}_{d}$ is a locally free sheaf of rank $d-g+1$ on the Jacobian scheme $J$.

Proof Let $y$ be a point on the scheme $J$, and let $s=h(y)$ be the image of the point $y$ under the structural morphism $h$ from $J$ to $S$. Then $y$ lies in the fiber $J_{S}$ of the morphism $h$, and if $X_{s}$ is the fiber of the structural morphism $f$ at $s$, then $J_{s}$ is the Jacobian of the smooth projective curve $X_{S}$ over the residue field $\kappa(s)$ at the point $s$. Denote by the same symbol $y$ the morphism from $\operatorname{Spec}(\kappa(y))$ to $J$, which corresponds to the point $y$, and by symbol $s^{\prime}$ the morphism from $\operatorname{Spec}(\kappa(y))$ to $S$, which factorises through the morphism from $\operatorname{Spec}(\kappa(s))$ to $S$, and the latter corresponds to the point $s$ on $S$. Then we obtain the following commutative diagram:

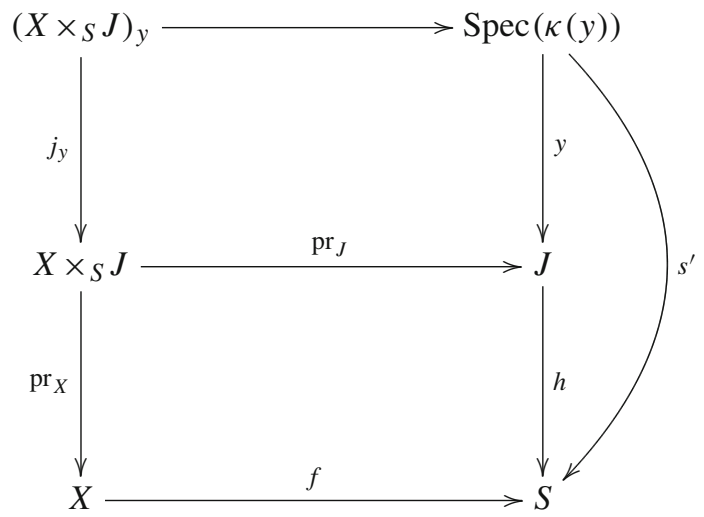

which shows that

$$
\left(X \times_{S} J\right)_{y}=X_{s^{\prime}},
$$

where

$$
X_{s^{\prime}}=X_{s} \times \operatorname{Spec}(\kappa(s)) \operatorname{Spec}(\kappa(y)) .
$$

Therefore, if

$$
\left(\mathcal{G}_{d}\right)_{y}=j_{y}^{*}\left(\mathcal{G}_{d}\right)
$$

is the pullback of the coherent sheaf $\mathcal{G}_{d}$ to the fibre $\left(X \times{ }_{S} J\right)_{y}$, we can also consider this pullback as a coherent sheaf on the smooth projective curve $X_{s^{\prime}}$ over the field $\kappa(y)$. Moreover, 


$$
\begin{aligned}
\left(\mathcal{G}_{d}\right)_{y} & =j_{y}^{*}\left(\mathcal{P} \otimes \operatorname{pr}_{X}^{*} \mathcal{O}_{X}(Z)^{\otimes d}\right) \\
& =j_{y}^{*}(\mathcal{P}) \otimes j_{y}^{*} \operatorname{pr}_{X}^{*}\left(\mathcal{O}_{X}(Z)^{\otimes d}\right)=j_{y}^{*}(\mathcal{P}) \otimes \mathcal{O}_{X_{s^{\prime}}}\left(Z_{s^{\prime}}\right)^{\otimes d},
\end{aligned}
$$

where $Z_{s^{\prime}}$ is the closed point on the curve $X_{s^{\prime}}$ induced by the point of intersection of $Z$ and $X_{s}$. Therefore,

$$
\operatorname{deg}\left(\left(\mathcal{G}_{d}\right)_{y}\right)=0+d=d
$$

Assume that

$$
d>2 g-2 .
$$

Then

$$
\operatorname{deg}\left(\left(\mathcal{G}_{d}\right)_{y}\right)>2 g-2,
$$

and, by the Riemann-Roch theorem,

$$
\operatorname{dim}_{\kappa(y)} H^{0}\left(\left(X \times_{S} J\right)_{y},\left(\mathcal{G}_{d}\right)_{y}\right)=H^{0}\left(X_{s^{\prime}},\left(\mathcal{G}_{d}\right)_{y}\right)=d-g+1 .
$$

As the function $y \mapsto \operatorname{dim}_{\kappa(y)} H^{0}\left(\left(X \times_{S} J\right)_{y},\left(\mathcal{G}_{d}\right)_{y}\right)$ is constant on $J$, by Grauert's theorem, the direct image

$$
\mathcal{E}_{d}=\operatorname{pr}_{J *} \mathcal{G}_{d}
$$

is locally free of rank $d-g+1$ on $J$, see [9, Chapter III, Corollary 12.9].

Consider the Cartesian square

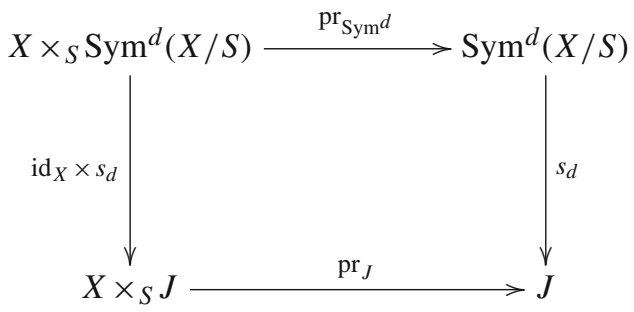

Here and below we write $\operatorname{pr}_{S^{\prime} m^{d}}$ instead of $\operatorname{pr}_{\operatorname{Sym}^{d}(X / S)}$ for shorter notation.

Lemma $2.2 s_{d}^{*} \mathcal{E}_{d}=\operatorname{pr}_{\mathrm{Sym}_{*}^{d}}\left(\mathrm{id}_{X} \times s_{d}\right)^{*} \mathcal{G}_{d}$.

Proof The structural morphism $f: X \rightarrow S$ is smooth, and hence flat, by assumption, and the morphism $J \rightarrow S$ is flat by [3, Theorem 1, p. 252]. The morphism $\operatorname{Sym}^{d}(X / S) \rightarrow S$ is also flat, see, for example, the arguments in [3, p. 253]. It follows that the morphism $s_{d}$ is flat. Therefore,

$$
s_{d}^{*} \mathcal{E}_{d}=s_{d}^{*} \operatorname{pr}_{J *} \mathcal{G}_{d}=\operatorname{pr}_{\operatorname{Sym}^{d}{ }_{*}}\left(\operatorname{id}_{X} \times s_{d}\right)^{*} \mathcal{G}_{d}
$$


by flat base change, see [9, Chapter III, Proposition 9.3] or [8, Proposition 1.4.15, p. 92 (436)].

Notice also that, since $\mathcal{E}_{d}$ is a locally free sheaf on the Jacobian $J$ by Lemma 2.1, so is its pullback $s_{d}^{*} \mathcal{E}_{d}$ on the symmetric power $\operatorname{Sym}^{d}(X / S)$.

Let now $D_{d}$ be the universal relative Cartier divisor on $X \times{ }_{S} \operatorname{Sym}^{d}(X / S)$. This divisor can be constructed as follows. Let $\Sigma_{d}$ be the $d$-th symmetric group permuting the elements $\{1, \ldots, d\}$. Permuting the elements $\{2, \ldots, d\}$ induces a group-theoretical embedding of $\Sigma_{d-1}$ into $\Sigma_{d}$. This embedding of groups induces the corresponding morphism on colimits,

$$
l_{0}: X \times{ }_{S} \operatorname{Sym}^{d-1}(X / S) \rightarrow \operatorname{Sym}^{d}(X / S) .
$$

Let then

$$
l: X \times{ }_{S} \operatorname{Sym}^{d-1}(X / S) \rightarrow X \times{ }_{S} \operatorname{Sym}^{d}(X / S)
$$

be a morphism uniquely defined by the projection $\operatorname{pr}_{X}$ and the morphism $l_{0}$. Then $l$ is a closed embedding, whose image is $D_{d}$.

The section $\sigma: S \rightarrow X$ induces the closed embedding

$$
\operatorname{Sym}^{d-1}(X / S) \rightarrow \operatorname{Sym}^{d}(X / S)
$$

whose image is a relative Cartier divisor $M_{d}$ on $\operatorname{Sym}^{d}(X / S)$ over $S$. Then

$$
M_{d}=\left(\sigma \times{ }_{S} \operatorname{id}_{\operatorname{Sym}^{d}(X / S)}\right) * D_{d}
$$

where

$$
\sigma \times{ }_{S} \operatorname{id}_{\operatorname{Sym}^{d}(X / S)}: S \times{ }_{S} \operatorname{Sym}^{d}(X / S) \rightarrow X \times{ }_{S} \operatorname{Sym}^{d}(X / S)
$$

and the pullback is the pullback of a Cartier divisor, see [18, Tag 01WV].

Let

$$
\mathcal{D}_{d}=\mathcal{O}_{X \times{ }_{S} \operatorname{Sym}^{d}(X / S)}\left(D_{d}\right) \quad \text { and } \quad \mathcal{M}_{d}=\mathcal{O}_{\operatorname{Sym}^{d}(X / S)}\left(M_{d}\right)
$$

be the invertible sheaves of the Cartier divisors $D_{d}$ and $M_{d}$ respectively.

The classes of the sheaves $\mathcal{D}_{d}$ and $\left(\mathrm{id}_{X} \times{ }_{S} s_{d}\right)^{*}\left(\mathcal{P} \otimes \mathrm{pr}_{X}^{*} \mathcal{O}_{X}(Z)^{\otimes d}\right)$ coincide modulo $\operatorname{pr}_{\text {Sym }^{d}}^{*} \operatorname{Pic}\left(\operatorname{Sym}^{d}(X / S)\right)$ inside the group $\mathcal{P i c}\left(X \times{ }_{S} \operatorname{Sym}^{d}(X / S)\right)$. Since $\mathcal{M}_{d}$ is in $\operatorname{Pic}\left(\operatorname{Sym}^{d}(X / S)\right)$, the same is true for $\mathcal{D}_{d}$ and the sheaf

$$
\mathcal{L}_{d}=\operatorname{pr}_{\text {Sym }^{d}}^{*} \mathcal{M}_{d} \otimes\left(\mathrm{id}_{X} \times{ }_{S} s_{d}\right)^{*}\left(\mathcal{P} \otimes \operatorname{pr}_{X}^{*} \mathcal{O}_{X}(Z)^{\otimes d}\right)
$$

on $X \times{ }_{S} \operatorname{Sym}^{d}(X / S)$. 
Lemma 2.3 There exists an invertible sheaf $\mathcal{K}$ on the base scheme $S$, such that

$$
\mathcal{D}_{d}=\mathcal{L}_{d} \otimes f^{*} \mathcal{K}
$$

on the scheme $X \times{ }_{S} \operatorname{Sym}^{d}(X / S)$.

Proof If $S$ is the spectrum of a field $k$, the section $\operatorname{Spec}(k) \rightarrow X$ gives us a point $x_{0}$ on $X$. The pullbacks of the divisors $D_{d}, X \times M_{d}, d\left(x_{0} \times \operatorname{Sym}^{d}(X)\right)$ and $\left(\operatorname{id}_{X} \times{ }_{S} s_{d}\right)^{*} P$ to $x_{0} \times \operatorname{Sym}^{d}(X)$ are equal to, respectively, the divisors $M_{d}, M_{d}, 0$ and 0 . Any point on $\operatorname{Sym}^{d}(X)$ can be identified with the corresponding effective divisor $D$ of degree $d$ on $X$. The pullbacks of the same divisors to $X \times D$ are, respectively, the divisors $D, 0, d x_{0}$ and $D-d x_{0}$. Therefore, the pullbacks of the line bundles $\mathcal{D}_{d}$ and $\mathcal{L}_{d}$ to $x_{0} \times \operatorname{Sym}^{d}(X)$ and $X \times D$ coincide, for any point $D$ on $\operatorname{Sym}^{d}(X)$. Applying the seesaw principle (see, for example, Section 5 in Chapter I in Milne's book [15]), we see that the line bundles $\mathcal{D}_{d}$ and $\mathcal{L}_{d}$ are isomorphic on the variety $X \times \operatorname{Sym}^{d}(X)$.

It follows that, in the general case, there exists an invertible sheaf $\mathcal{K}$ on the base scheme $S$ such that $\mathcal{D}_{d}=\mathcal{L}_{d} \otimes f^{*} \mathcal{K}$, see [10, Proposition 1.2, p. 54].

Lemma 2.4 $\mathrm{pr}_{\mathrm{Sym}_{*}^{d}} \mathcal{D}_{d}=\mathcal{M}_{d} \otimes s_{d}^{*} h^{*} \mathcal{K} \otimes s_{d}^{*} \varepsilon_{d}$.

Proof Using Lemma 2.3 and the definition of the sheaf $\mathcal{L}_{d}$, we obtain

$$
\mathcal{D}_{d}=\operatorname{pr}_{\mathrm{Sym}^{d}}^{*} \mathcal{M}_{d} \otimes\left(\mathrm{id}_{X} \times{ }_{S} s_{d}\right)^{*}\left(\mathcal{P} \otimes \operatorname{pr}_{X}^{*} \mathcal{O}_{X}(Z)^{\otimes d} \otimes \operatorname{pr}_{X}^{*} f^{*} \mathcal{K}\right),
$$

or, equivalently,

$$
\mathcal{D}_{d}=\operatorname{pr}_{\text {Sym }^{d}}^{*} \mathcal{M}_{d} \otimes\left(\mathrm{id}_{X} \times s_{d}\right)^{*} \mathcal{G}_{d} \otimes\left(\operatorname{id}_{X} \times{ }_{S} s_{d}\right)^{*} \operatorname{pr}_{X}^{*} f^{*} \mathcal{K}
$$

on the scheme $X \times{ }_{S} \operatorname{Sym}^{d}(X / S)$. Using the projection formula and Lemma 2.2, we calculate:

$$
\begin{aligned}
\operatorname{pr}_{\operatorname{Sym}^{d}} \mathcal{D}_{d} & =\operatorname{pr}_{\operatorname{Sym}^{d}}\left(\operatorname{pr}_{\operatorname{Sym}^{d}}^{*} \mathcal{M}_{d} \otimes\left(\operatorname{id}_{X} \times s_{d}\right)^{*} \mathcal{G}_{d} \otimes\left(\mathrm{id}_{X} \times s_{d}\right)^{*} \operatorname{pr}_{X}^{*} f^{*} \mathcal{K}\right) \\
& =\operatorname{pr}_{\operatorname{Sym}^{d}}\left(\operatorname{pr}_{\operatorname{Sym}^{d}}^{*} \mathcal{M} \mathcal{M}_{d} \otimes\left(\mathrm{id}_{X} \times s_{d}\right)^{*} \mathcal{G}_{d} \otimes \operatorname{pr}_{\operatorname{Sym}^{d}}^{*} s_{d}^{*} h^{*} \mathcal{K}\right) \\
& =\operatorname{pr}_{\operatorname{Sym}^{d}}\left(\operatorname{pr}_{\operatorname{Sym}^{d}}^{*}\left(\mathcal{M}_{d} \otimes s_{d}^{*} h^{*} \mathcal{K}\right) \otimes\left(\operatorname{id}_{X} \times s_{d}\right)^{*} \mathcal{G}_{d}\right) \\
& =\mathcal{M}_{d} \otimes s_{d}^{*} h^{*} \mathcal{K} \otimes \operatorname{pr}_{\operatorname{Sym}^{d}}\left(\left(\operatorname{id}_{X} \times s_{d}\right)^{*} \mathcal{G}_{d}\right) \\
& =\mathcal{M}_{d} \otimes s_{d}^{*} h^{*} \mathcal{K} \otimes s_{d}^{*} \mathcal{E}_{d} .
\end{aligned}
$$

For shorter notation, let

$$
\mathcal{E}_{d}^{\prime}=h^{*} \mathcal{K} \otimes \mathcal{E}_{d}
$$

be the relative Picard bundle amended by the invertible sheaf $h^{*} \mathcal{K}$, and let

$$
\mathcal{Q}_{d}=s_{d}^{*} h^{*} \mathcal{K} \otimes s_{d}^{*} \mathcal{E}_{d}=s_{d}^{*}\left(\mathcal{E}_{d}^{\prime}\right)
$$


Lemma $2.5 \mathrm{pr}_{\mathrm{Sym}^{d}{ }_{*}} \mathcal{D}_{d}=\mathcal{M}_{d} \otimes \mathcal{Q}_{d}$.

Proof This is a reformulation of Lemma 2.4.

Below, for any $\mathcal{O}_{T}$-module $\mathcal{F}$, on a ringed space $\left(T, \mathcal{O}_{T}\right)$, we denote by $\breve{\mathcal{F}}$ the module dual to $\mathcal{F}$.

Proposition 2.6 We have the following two key identifications:

$$
\operatorname{pr}_{\operatorname{Sym}^{d}} \mathcal{D}_{d}=\mathcal{H} \operatorname{Hom}\left(\check{Q}_{d}, \mathcal{M}_{d}\right)
$$

and

$$
H^{0}\left(X \times{ }_{S} \operatorname{Sym}^{d}(X / S), \mathcal{D}_{d}\right)=\operatorname{Hom}\left(\check{Q}_{d}, \mathcal{M}_{d}\right)
$$

Proof The pullback of a locally free sheaf is locally free. Since $\varepsilon_{d}$ is locally free by Lemma 2.1, and $\mathcal{K}$ is invertible, the sheaf $\mathcal{E}_{d}^{\prime}$ is locally free. Then $\mathcal{Q}_{d}$ is a locally free sheaf on the $d$-th symmetric power $\operatorname{Sym}^{d}(X / S)$. Therefore, the double dual sheaf $\check{\mathscr{Q}}_{d}$ can be identified with the original sheaf $\mathcal{Q}_{d}$ on $\operatorname{Sym}^{d}(X / S)$. Applying Lemma 2.5, we obtain the first identification

$$
\operatorname{pr}_{\operatorname{Sym}^{d}} \mathcal{D}_{d}=\mathcal{M}_{d} \otimes \check{\check{Q}}_{d}=\mathcal{H} \operatorname{Hom}\left(\check{\mathscr{Q}}_{d}, \mathcal{M}_{d}\right)
$$

As the group of global sections of the sheaf $\operatorname{pr}_{\operatorname{Sym}^{d}} \mathcal{D}_{d}$ on $\operatorname{Sym}^{d}(X / S)$ is the same as the group of global sections of the sheaf $\mathcal{D}_{d}$ on $X \times{ }_{S} \operatorname{Sym}^{d}(X / S)$, and the group of global sections of the sheaf $\mathcal{H o m}\left(\check{Q}_{d}, \mathcal{M}_{d}\right)$ is the group $\operatorname{Hom}\left(\check{Q}_{d}, \mathcal{M}_{d}\right)$, the first identification implies the second one:

$$
H^{0}\left(X \times{ }_{S} \operatorname{Sym}^{d}(X / S), \mathcal{D}_{d}\right)=H^{0}\left(\operatorname{Sym}^{d}(X / S), \operatorname{pr}_{\operatorname{Sym}^{d}} \mathcal{D}_{d}\right)=\operatorname{Hom}\left(\check{\mathcal{Q}}_{d}, \mathcal{M}_{d}\right) .
$$

Now we are ready to prove Theorem A from the introduction.

Let first $\left(T, \mathcal{O}_{T}\right)$ be an arbitrary locally ringed topological space, let $\mathcal{F}$ be a locally free $\mathcal{O}_{T}$-module of finite rank $r$, and let $\mathcal{L}$ be an invertible $\mathcal{O}_{T}$-module on $T$. A morphism of $\mathcal{O}_{T}$-modules

$$
q: \mathcal{F} \rightarrow \mathcal{L}
$$

is an epimorphism if and only if for any point $t \in T$ the induced homomorphism

$$
q_{t}: \mathcal{F}_{t}=\mathcal{O}_{t}^{\oplus r} \rightarrow \mathcal{O}_{t}=\mathcal{L}_{t}
$$

on stalks is surjective. The latter is equivalent to saying that, after the obvious identification $\operatorname{Hom}_{\mathcal{O}_{t}}\left(\mathcal{O}_{t}^{\oplus r}, \mathcal{O}_{t}\right)=\mathcal{O}_{t}^{\oplus r}$, the morphism $q_{t}$ corresponds to the $r$-tuple $\left(\phi_{1}, \ldots\right.$, $\left.\phi_{r}\right)$ such that the function $\phi_{i}$ is not in the maximal ideal of the local ring $\mathcal{O}_{t}$ at least for one index $i$. 
We are going to apply this simple observation in our case. Namely, consider an arbitrary morphism $q: \breve{Q}_{d} \rightarrow \mathcal{M}_{d}$ of $\mathcal{O}_{\operatorname{Sym}^{d}(X / S)}$-modules on the symmetric power $\operatorname{Sym}^{d}(X / S)$, and let $q^{\prime}$ be the global section corresponding to $q$ under the second identification of Proposition 2.6. Then $q^{\prime}$ is a section in $H^{0}\left(\operatorname{Sym}^{d}(X / S), \operatorname{pr}_{\operatorname{Sym}^{d}} \mathcal{D}_{d}\right)$, and the latter group coincides with the group $H^{0}\left(X \times{ }_{S} \operatorname{Sym}^{d}(X / S), \mathcal{D}_{d}\right)$. Let $r$ be the rank of the sheaf $\check{Q}_{d}$, and suppose that $q$ is not an epimorphism. According to the observation above, there exists a point $t$ on the scheme $\operatorname{Sym}^{d}(X / S)$, such that, if $\left(\phi_{1}, \ldots, \phi_{r}\right)$ are the regular functions representing the germ of $q$ at $t$, then $\phi_{i}(t)=0$ for all indices $i=1, \ldots, r$. Taking into account the identifications of Proposition 2.6, we see that the germ of the global section $q^{\prime}$ at $t$ has the same property, i.e., if $\left(\phi_{1}^{\prime}, \ldots, \phi_{r}^{\prime}\right)$ are the regular functions representing the germ of $q^{\prime}$ at $t$, then $\phi_{i}^{\prime}(t)=0$ for all indices $i=1, \ldots, r$. It follows that the composition $\phi_{i}^{\prime} \circ \mathrm{pr}_{\mathrm{Sym}^{d}}$ is regular in a neigbourhood of the image of the pullback $X \times{ }_{S} t$ in $X \times{ }_{S} \operatorname{Sym}^{d}(X / S)$ and vanishes at all points of this image. But this is not possible if the section $q^{\prime}$, as an element of the group $H^{0}\left(X \times{ }_{S} \operatorname{Sym}^{d}(X / S), \mathcal{D}_{d}\right)$, is the canonical section, i.e., the local equation $1_{D_{d}}$ of the Cartier divisor $D_{d}$ on the scheme $X \times{ }_{S} \operatorname{Sym}^{d}(X / S)$, in terms of [18, Tag $0 \mathrm{C} 4 \mathrm{~S}]$. Therefore, if the morphism $q$ corresponds to the section $1_{D_{d}}$, in which case we should write $q=q_{D_{d}}$, then $q_{D_{d}}$ is an epimorphism of sheaves from $\check{\mathscr{Q}}_{d}$ onto $\mathcal{M}_{d}$.

For shorter notation, let

$$
\mathcal{F}_{d}=\check{\mathcal{E}}_{d}^{\prime}
$$

be the dual to the amended Picard sheaf. Since $\check{Q}_{d}$ is $s_{d}^{*}\left(\mathcal{F}_{d}\right)$, in new terms the morphism $q_{D_{d}}$ is an epimorphism

$$
q_{D_{d}}: s_{d}^{*}\left(\mathcal{F}_{d}\right) \rightarrow \mathcal{M}_{d}
$$

of $\mathcal{O}_{\operatorname{Sym}^{d}(X / S)}$-modules on the scheme $\operatorname{Sym}^{d}(X / S)$. This yields the corresponding morphism

$$
r_{d}: \operatorname{Sym}^{d}(X / S) \rightarrow \mathbb{P}\left(\mathcal{F}_{d}\right),
$$

such that the diagram

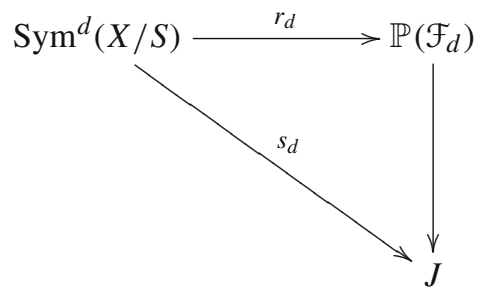

commutes, and

$$
r_{d}^{*} \mathcal{O}(1)=\mathcal{M}_{d},
$$


see, for example, [9, Chapter II, Proposition 7.12].

All three morphisms in the latter commutative triangle are over the base $S$. The specialisation of the morphism $r_{d}$ to each point in $S$ naturally coincides with the morphism from [17, Proposition 11]. Since $d>2 g-2$, the map $r_{d}$ is an isomorphism fiberwise, see loc. cit. In particular, it is an isomorphism over the generic point $\eta$ of the integral scheme $S$. Therefore, there exists a Zariski open $U$ in $S$ such that the restricted morphism

$$
\left(r_{d}\right)_{U}: X_{U} \rightarrow \mathbb{P}\left(\mathcal{F}_{d}\right)_{U}=\mathbb{P}\left(\left.\mathcal{F}_{d}\right|_{U}\right)
$$

is an isomorphism over $J_{U}$, where for a scheme $Y$ over $S$ we denote by $Y_{U} \rightarrow U$ the pullback of the structural morphism $Y \rightarrow S$ to $U$. Then $r_{d}$ is a birational morphism from $\operatorname{Sym}^{d}(X / S)$ to $\mathbb{P}\left(\mathcal{F}_{d}\right)$.

Since $X$ is projective over $S$, the structural morphism $f: X \rightarrow S$ is proper. It follows that the structural morphism from $\operatorname{Sym}^{d}(X / S)$ to $S$ is proper. Since the morphism $h: J \rightarrow S$ is separated, the morphism $s_{d}: \operatorname{Sym}^{d}(X / S) \rightarrow J$ is proper by [9, Corollary 4.8, p. 102]. The projective bundle morphism $\mathbb{P}\left(\mathcal{F}_{d}\right) \rightarrow J$ is separated as well. Applying the same principle, we obtain that the morphism $r_{d}: \operatorname{Sym}^{d}(X / S) \rightarrow \mathbb{P}\left(\mathcal{F}_{d}\right)$ is proper.

Next, the morphism $r_{d}$ induces a bijection on fibres at every point $s$ on the scheme $S$. It follows that $r_{d}$ is a bijection on points, and, therefore, it is a homeomorphism of the topological spaces underlying the schemes in question. Then $r_{d}$ is an affine morphism of schemes, see, for example, [18, Tag 04DE]. Since $r_{d}$ is affine and proper, it is a finite morphism of schemes (it is obvious, but see [18, Tag 01WN] if necessary).

Finally, if we assume, in addition, that the base scheme $S$ is normal, then the projective bundle $\mathbb{P}\left(\mathcal{F}_{d}\right)$ is normal as well, and the morphism $r_{d}$ is an isomorphism by the Zariski Main Theorem, see, for example, [18, Tag 0AB1]. This finishes the proof of Theorem A.

\section{The proof of Theorem B and Corollary C}

Let $R$ be a Dedekind domain. For any scheme $X$ of finite type and separated over $\operatorname{Spec}(R)$ we have an appropriate intersection theory on $X$, see [6, Sections 20.1 and 20.2]. Assuming that $X$ is equidimensional, let $C H^{i}(X)$ be the Chow group of codimension $i$ algebraic cycles with coefficients in $\mathbb{Q}$ modulo rational equivalence on $X$. If $Z$ is a closed subscheme in $X$ then $[Z]$ is the corresponding fundamental class in the Chow group of $X$.

Fix an integral regular scheme $S$ of finite type and separated over $\operatorname{Spec}(R)$. We will be working with schemes $X$ which are smooth and projective over $S$. Notice that, since projective morphisms are proper and smooth morphisms are flat, the structural morphism of $X$ over $S$ is flat and proper. In particular, we can push algebraic cycles forward and pull them back, with regard to the structural morphism from $X$ to $S$. The same is true with regard to various projections we will be using in our computations below. If $X$ and $Y$ are two schemes, both smooth and projective over $S$, we let $X_{j}$ be the connected components of the scheme $X$, and for any non-negative $m$ we set 


$$
C H_{S}^{m}(X, Y)=\bigoplus_{j} C H^{e_{j}+m}\left(X_{j} \times{ }_{S} Y\right)
$$

be the group of relative correspondences of degree $m$ from $X$ to $Y$ over $S$, where $e_{j}$ is the relative dimension of $X_{j}$ over $S$. As in the absolute case, for any two correspondences

$$
\Sigma \in C H_{S}^{m}(X, Y) \quad \text { and } \quad \Theta \in C H_{S}^{n}(Y, Z)
$$

their composition $\Theta \circ \Sigma$ is defined by the formula

$$
\Theta \circ \Sigma=\operatorname{pr}_{13 *}\left(\operatorname{pr}_{12}^{*}(\Sigma) \cdot \operatorname{pr}_{23}^{*}(\Theta)\right)
$$

where the central dot denotes the intersection of cycle classes in the sense of [6], and $\mathrm{pr}_{i j}$ are the projections.

Let us stress once again that this all works well over $S$ due to the intersection theory on schemes which are separated and of finite type over a regular scheme, developed in Sections 20.1 and 20.2 of Fulton's book [6] (see also [12, Remark 1.1, p. 367]).

The category $\mathrm{M}(S)$ of Chow motives over $S$ with coefficients in $\mathbb{Q}$ can be defined as a pseudoabelian envelope of the category of correspondences with certain "Tate twists" indexed by integers. Then objects in $\mathrm{M}(S)$ are triples $(X, \Sigma, m)$ consisting of a smooth projective scheme $X$ over $S$, a relative idempotent

$$
\Sigma \in C H_{S}^{0}(X, X)
$$

and an integer $m$. The Hom-groups ( $\mathbb{Q}$-vector spaces, actually) are given by the formula

$$
\operatorname{Hom}_{M(S)}((X, \Sigma, m),(Y, \Theta, n))=\Theta \circ C H_{S}^{n-m}(X, Y) \circ \Sigma .
$$

The category $\mathrm{M}(S)$ is tensor, rigid and $\mathbb{Q}$-linear, with tensor unit

$$
\mathbb{1}=\left(S, \Delta_{S / S}, 0\right)
$$

and the Lefschetz motive

$$
\mathbb{L}=\left(S, \Delta_{S / S},-1\right) .
$$

If $X$ is a smooth projective scheme of relative dimension $e$ over $S$, and if $Z$ a multisection of degree $m$ of the structural morphism $X \rightarrow S$, the relative correspondences

$$
\pi_{0}=\frac{1}{m} \cdot\left[Z \times_{S} X\right] \quad \text { and } \quad \pi_{2 e}=\frac{1}{m} \cdot\left[X \times{ }_{S} Z\right]
$$

determine the motives $\mathbb{1}$ and $\mathbb{L}$ up to an isomorphism.

Below we will be also freely using the results and terminology from the theory of finite-dimensional objects in rigid tensor categories, which can be found either in $[2$, Chapter II] or in [1, Chapter 12]. 
Let $f: X \rightarrow S$ be a smooth projective scheme of relative dimension $e$ over the base $S$. Let $\eta$ be the generic point of $S$, and assume that the generic fibre $X_{\eta}$ of the structural morphism $f$ has a rational point over $\eta$. Then there exists a Zariski open subset $U \subset S$, and a section $\sigma_{U}: U \rightarrow X_{U}$ of the structural morphism

$$
f_{U}: X_{U}=X \times{ }_{S} U \rightarrow U
$$

Let $Z$ be the image of the section $\sigma_{U}$ in $X_{U}$, and let $\bar{Z}$ be the Zariski closure of $Z$ in $X$. Then $\bar{Z}$ is a prime cycle of degree one over $S$, and we consider two relative projectors

$$
\bar{\pi}_{0}=\left[\bar{Z} \times{ }_{S} X\right] \quad \text { and } \quad \bar{\pi}_{2 e}=\left[X \times_{S} \bar{Z}\right]
$$

of the motive $M(X)$. If the self-intersection of the cycle class $[\bar{Z}]$ is 0 then $\bar{\pi}_{0}$ and $\bar{\pi}_{2}$ are orthogonal in the associative ring $C H_{S}^{0}(X, X)$, in which case we can use them in order to split $\mathbb{1}_{S}$ and $\mathbb{L}_{S}^{\otimes e}$ from $M(X)$ simultaneously. But if $[\bar{Z}] \cdot[\bar{Z}] \neq 0$, then the projectors $\bar{\pi}_{0}$ and $\bar{\pi}_{2}$ are not orthogonal, and we need to modify them.

In what follows, for any natural number $n$, let

$$
f_{n}: X \times{ }_{S} \cdots \times{ }_{S} X \rightarrow S
$$

be the structural morphism of the $n$-fold fibred product over the base $S$, and always write $f$ instead of $f_{1}$. Let, furthermore,

$$
\zeta=f_{*}([\bar{Z}] \cdot[\bar{Z}])
$$

be the push-forward of the self-intersection of the class of $\bar{Z}$ to the base $S$, and consider the pullback

$$
\theta=f_{2}^{*}(\zeta)
$$

with regard to the structural morphism $f_{2}$ from $X \times{ }_{S} X$ to $S$. The cycle class $\theta$ can be considered as a vertical correcting term for the projector $\bar{\pi}_{0}$ in the following sense.

Lemma 3.1 The projectors $\tau_{0}=\bar{\pi}_{0}-\theta$ and $\bar{\pi}_{2 e}$ are pairwise orthogonal.

Proof For any indices $i$ and $j$, let

$$
\operatorname{pr}_{i j}: X \times{ }_{S} \cdots \times{ }_{S} X \rightarrow X \times{ }_{S} X \quad \text { and } \quad \operatorname{pr}_{i}: X \times{ }_{S} \cdots \times_{S} X \rightarrow X
$$

be the projections corresponding to their indexes. Since $f_{2} \mathrm{pr}_{i j}=f_{3}$, one has

$$
\operatorname{pr}_{i j}^{*}(\theta)=\operatorname{pr}_{i j}^{*} f_{2}^{*}(\zeta)=f_{3}^{*}(\zeta)
$$


Therefore,

$$
\begin{aligned}
\theta \circ \theta & =\operatorname{pr}_{13 *}\left(\operatorname{pr}_{12}^{*}(\theta) \cdot \operatorname{pr}_{23}^{*}(\theta)\right)=\operatorname{pr}_{13 *} f_{3}^{*}(\zeta \cdot \zeta)=\operatorname{pr}_{13 *} \operatorname{pr}_{13}^{*} f_{2}^{*}(\zeta \cdot \zeta) \\
& =f_{2}^{*}(\zeta \cdot \zeta) \cdot \operatorname{pr}_{13 *}\left(\left[X \times{ }_{S} X \times{ }_{S} X\right]\right)=f_{2}^{*}(\zeta \cdot \zeta) \cdot 0=0 .
\end{aligned}
$$

For projections from $X \times_{S} X \times{ }_{S} X$, since $\mathrm{pr}_{1} \mathrm{pr}_{23}=\mathrm{pr}_{2}$, we get

$$
\operatorname{pr}_{23}^{*}\left(\bar{\pi}_{0}\right)=\operatorname{pr}_{23}^{*} \operatorname{pr}_{1}^{*}([\bar{Z}])=\operatorname{pr}_{2}^{*}([\bar{Z}]) \text {. }
$$

Then compute

$$
\begin{aligned}
\bar{\pi}_{0} \circ \theta & =\operatorname{pr}_{13 *}\left(\operatorname{pr}_{12}^{*}(\theta) \cdot \operatorname{pr}_{23}^{*}\left(\bar{\pi}_{0}\right)\right)=\operatorname{pr}_{13 *}\left(f_{3}^{*}(\zeta) \cdot \operatorname{pr}_{2}^{*}([\bar{Z}])\right) \\
& =\operatorname{pr}_{13 *}\left(\operatorname{pr}_{2}^{*} f_{1}^{*}(\zeta) \cdot \operatorname{pr}_{2}^{*}([\bar{Z}])\right)=\operatorname{pr}_{13 *} \operatorname{pr}_{2}^{*}\left(f_{1}^{*}(\zeta) \cdot[\bar{Z}]\right) \\
& =f_{2}^{*} f_{1 *}\left(f_{1}^{*}(\zeta) \cdot[\bar{Z}]\right)=f_{2}^{*}\left(\zeta \cdot f_{1 *}([\bar{Z}])\right) \\
& =f_{2}^{*}(\zeta \cdot[S])=f_{2}^{*}(\zeta)=\theta .
\end{aligned}
$$

The transposition of the cycle class $\theta$ is $\theta$, and the transposition of the cycle class $\bar{\pi}_{0}$ is $\bar{\pi}_{2 e}$. Therefore, the last equality yields

$$
\theta \circ \bar{\pi}_{2 e}=\theta \text {. }
$$

Since $\bar{\pi}_{2 e}=\left[X \times{ }_{S} \bar{Z}\right]$, we have that

$$
\begin{aligned}
\bar{\pi}_{2 e} \circ \theta & =\operatorname{pr}_{13 *}\left(\operatorname{pr}_{12}^{*}(\theta) \cdot \operatorname{pr}_{23}^{*}\left(\bar{\pi}_{2 e}\right)\right)=\operatorname{pr}_{13 *}\left(f_{3}^{*}(\zeta) \cdot \operatorname{pr}_{3}^{*}([\bar{Z}])\right) \\
& =\operatorname{pr}_{13 *}\left(\operatorname{pr}_{3}^{*} f_{1}^{*}(\zeta) \cdot \operatorname{pr}_{3}^{*}([\bar{Z}])\right)=\operatorname{pr}_{13 *} \operatorname{pr}_{3}^{*}\left(f_{1}^{*}(\zeta) \cdot[\bar{Z}]\right)=0
\end{aligned}
$$

because $\operatorname{pr}_{13 *} \operatorname{pr}_{3}^{*}=0$ in general. Transposing the cycles we get

$$
\theta \circ \bar{\pi}_{0}=0 .
$$

Finally,

$$
\begin{aligned}
\bar{\pi}_{0} \circ \bar{\pi}_{2 e} & =\operatorname{pr}_{13 *}\left(\operatorname{pr}_{12}^{*}\left(\bar{\pi}_{2 e}\right) \cdot \operatorname{pr}_{23}^{*}\left(\bar{\pi}_{0}\right)\right)=\operatorname{pr}_{13 *}\left(\operatorname{pr}_{12}^{*} \operatorname{pr}_{2}^{*}([\bar{Z}]) \cdot \operatorname{pr}_{23}^{*} \operatorname{pr}_{1}^{*}([\bar{Z}])\right) \\
& =\operatorname{pr}_{13 *} \operatorname{pr}_{2}^{*}([\bar{Z}] \cdot[\bar{Z}])=f_{2}^{*} f_{1 *}([\bar{Z}] \cdot[\bar{Z}])=f_{2}^{*}(\zeta)=\theta
\end{aligned}
$$

and

$$
\begin{aligned}
\bar{\pi}_{2 e} \circ \bar{\pi}_{0} & =\operatorname{pr}_{13 *}\left(\operatorname{pr}_{12}^{*}\left(\bar{\pi}_{0}\right) \cdot \operatorname{pr}_{23}^{*}\left(\bar{\pi}_{2 e}\right)\right)=\operatorname{pr}_{13 *}\left(\operatorname{pr}_{12}^{*} \operatorname{pr}_{1}^{*}([\bar{Z}]) \cdot \operatorname{pr}_{23}^{*} \operatorname{pr}_{2}^{*}([\bar{Z}])\right) \\
& \left.=\operatorname{pr}_{13 *}\left(\operatorname{pr}_{1}^{*}([\bar{Z}]) \cdot \operatorname{pr}_{3}^{*}([\bar{Z}])\right)=\operatorname{pr}_{13 *}\left(\left[\bar{Z} \times{ }_{S} X \times{ }_{S} \bar{Z}\right]\right)\right)=0 .
\end{aligned}
$$

Now, using the obtained equalities, one can easily compute

$$
\begin{aligned}
\tau_{0} \circ \tau_{0} & =\left(\bar{\pi}_{0}-\theta\right) \circ\left(\bar{\pi}_{0}-\theta\right) \\
& =\bar{\pi}_{0} \circ \bar{\pi}_{0}-\bar{\pi}_{0} \circ \theta-\theta \circ \bar{\pi}_{0}+\theta \circ \theta=\bar{\pi}_{0}-\theta-0+0=\tau_{0}, \\
\tau_{0} \circ \bar{\pi}_{2 e} & =\left(\bar{\pi}_{0}-\theta\right) \circ \bar{\pi}_{2 e}=\bar{\pi}_{0} \circ \bar{\pi}_{2 e}-\theta \circ \bar{\pi}_{2 e}=\theta-\theta=0
\end{aligned}
$$


and

$$
\bar{\pi}_{2 e} \circ \tau_{0}=\bar{\pi}_{2 e} \circ\left(\bar{\pi}_{0}-\theta\right)=\bar{\pi}_{2 e} \circ \bar{\pi}_{0}-\bar{\pi}_{2 e} \circ \theta=0-0=0 .
$$

Now, setting

$$
\tilde{\pi}=\Delta_{X / S}-\tau_{0}-\bar{\pi}_{2},
$$

since $\tau_{0}$ and $\bar{\pi}_{2}$ are orthogonal by Lemma 3.1, we obtain that all the projectors $\tau_{0}, \tilde{\pi}$ and $\bar{\pi}_{2 e}$ are pair-wise orthogonal. Let then

$$
A=\left(X, \tau_{0}, 0\right) \quad \text { and } \quad \tilde{M}(X)=(X, \tilde{\pi}, 0)
$$

be the motives given by the projectors $\tau_{0}$ and $\tilde{\pi}$ respectively. The projector $\bar{\pi}_{2 e}$ defines the $e$-th tensor power of relative Lefschetz motive $\mathbb{L}_{S}$. Then we obtain the splitting

$$
M(X)=A \oplus \tilde{M}(X) \oplus \mathbb{L}_{S}^{\otimes e}
$$

Certainly, one can split the same motive $M(X)$ in other way around by setting

$$
\tau_{2 e}=\bar{\pi}_{2 e}-\theta, \quad \widehat{\pi}=\Delta_{X / S}-\bar{\pi}_{0}-\tau_{2 e},
$$

and proving a lemma similar to Lemma 3.1. Then we obtain that $\bar{\pi}_{0}, \widehat{\pi}$ and $\tau_{2 e}$ are pair-wise orthogonal idempotents in the ring $C H_{S}^{0}(X, X)$, so that, if $B$ and $\widehat{M}(X)$ are the motives given by the projectors $\tau_{2 e}$ and $\widehat{\pi}$ respectively, then

$$
M(X)=\mathbb{1}_{S} \oplus \widehat{M}(X) \oplus B .
$$

Lemma $3.2 A \simeq \mathbb{1}$ and $B \simeq \mathbb{L}^{\otimes e}$.

Proof The cycle class $\zeta$ is represented by a linear combination of some closed subschemes $S_{i}$ in $S$, say

$$
\sum_{i=1}^{m} n_{i} S_{i}
$$

Then

$$
\theta=\sum_{i=1}^{m} n_{i}\left[V_{i} \times{ }_{S_{i}} V_{i}\right],
$$

where $V_{i}=X \times{ }_{S} S_{i}$ for each index $i$. Consider the correspondences

$$
a=[X] \in C H_{S}^{-e}(X, S)=C H^{0}(X)
$$


and

$$
b=[\bar{Z}]-\sum_{i=1}^{m} n_{i}\left[V_{i}\right] \in C H_{S}^{e}(S, X)=C H^{e}(X) .
$$

Then it is easy to compute:

$$
b \circ a=\operatorname{pr}_{13 *}\left(\operatorname{pr}_{12}^{*}(a) \cdot \operatorname{pr}_{23}^{*}(b)\right)=\left[X \times{ }_{S} \bar{Z}\right]-\sum_{i=1}^{m} n_{i}\left[V_{i} \times S_{i} V_{i}\right]=\tau_{2 e}
$$

and

$$
a \circ b=\operatorname{pr}_{13 *}\left(\operatorname{pr}_{12}^{*}(b) \cdot \operatorname{pr}_{23}^{*}(a)\right)=f_{1 *}(b)=\Delta_{S / S} .
$$

Lemma 3.2 allows us to identify both splittings and define the decomposition

$$
M(X)=\mathbb{1} \oplus M^{1}(X) \oplus \mathbb{L}^{\otimes e},
$$

in which $M^{1}(X)$ is either $\tilde{M}$ or $\widehat{M}$.

Now we start proving Theorem B. The properties of finite-dimensional objects guarantee that we only need to prove it for the motives of relative curves. Therefore, as in the previous section, we now assume that $e=1$, and that the fibres of the morphism $f: X \rightarrow S$ are connected. Secondly, without loss of generality, one can assume that the generic fibre $X_{\eta}$ has a point rational over $\eta$, so that the morphism $f_{U}: X_{U} \rightarrow U$ has a section $\sigma_{U}: U \rightarrow X_{U}$, for some nonempty Zariski open subset $U$ in $S$, and therefore Theorem A applies to the relative curve $f_{U}: X_{U} \rightarrow U$ with the section $\sigma_{U}$.

As above, let $g$ be the genus of the fibres of the morphism $f: X \rightarrow S$.

Proposition 3.3 For any integer $d>2 g-2$ there exists a nonempty Zariski closed subset $U$ in $S$ such that

$$
\operatorname{Sym}^{d}\left(M^{1}\left(X_{U}\right)\right)=0
$$

in $\mathrm{M}(U)$.

Proof For short, let $Y=X_{U}$, and let $Z$ be the image of $U$ under the section $\sigma_{U}$ in $Y$. Without loss of generality, one can shrink $U$ to have $[Z] \cdot[Z]=0$ in $C H_{U}^{0}(Y, Y)$. In such a case the correspondences $\pi_{0}=\left[Z \times{ }_{U} Y\right]$ and $\pi_{2}=\left[Y \times{ }_{U} Z\right]$ are pair-wise orthogonal. Define the middle projector for $Y / U$ by setting $\pi_{1}=\Delta_{Y / U}-\pi_{0}-\pi_{2}$.

Consider the canonical morphism

$$
t_{d}:(Y / U)^{d} \rightarrow \operatorname{Sym}^{d}(Y / U)
$$

from the $d$-fold fibred product onto the symmetric power. This morphism is obviously proper. Following Kimura, we will show now that the cycle class $\left(t_{d} \times{ }_{S} t_{d}\right)_{*}\left(\pi_{1}\right)$ is 0 in the Chow group $C H_{U}^{0}\left(\operatorname{Sym}^{d}(Y / U), \operatorname{Sym}^{d}(Y / U)\right)$.

To illustrate the arguments we will be using the following diagram: 


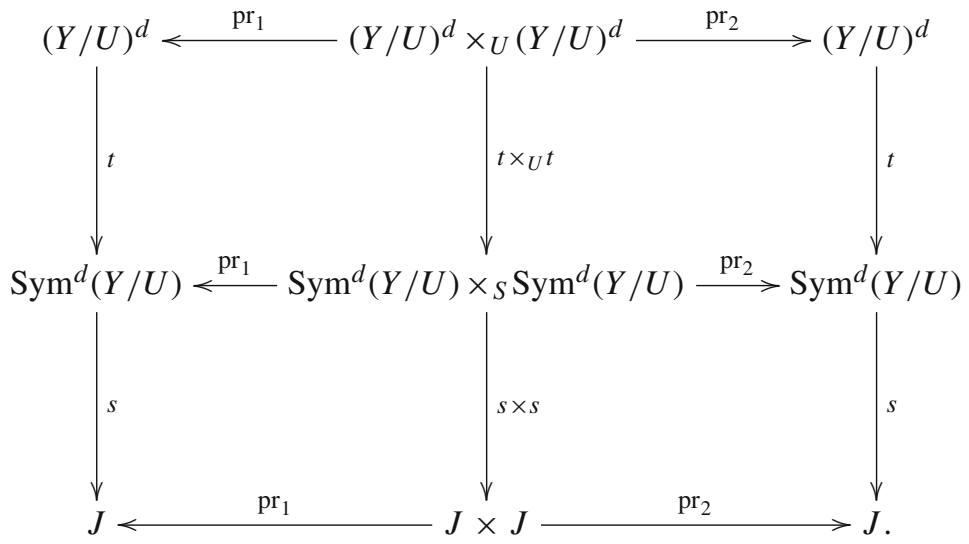

Here $J$ is the Jacobian scheme of the relative curve $Y / U, t=t_{d}$ and $s=s_{d}$ for shorter notation.

For any scheme $V$, smooth and projective over $S$, the Chern character homomorphism

$$
\operatorname{ch}: K(V) \otimes \mathbb{Q} \rightarrow C H^{*}(V) \otimes \mathbb{Q}
$$

from the $K$-theory of $V$ to the rational Chow algebra is still an isomorphism, see the comment in [6, p. 393]. The same applies to the projective bundle $\mathbb{P}(\mathcal{E})$ on $V$, where $\mathcal{E}$ is a locally free sheaf on $V$. Then, by [19, Theorem 1.1, p. 365], we have that the projective bundle formula holds true in our context as well, i.e., $C H^{*}(\mathbb{P}(\mathcal{E}))$ is a free algebra over $C H^{*}(V)$ generated by the powers of $\mathcal{O}(1)$.

Now, applying the projective bundle formula in our local case over $U$, we obtain that the pullback homomorphism

$$
s^{*}: C H^{*}(J) \rightarrow C H^{*}\left(\operatorname{Sym}^{d}(Y / U)\right)
$$

turns the group $C H^{*}\left(\operatorname{Sym}^{d}(Y / U)\right)$ in to a free module over the commutative ring $C H^{*}(J)$, generated by the cycle classes

$$
1, H, H^{2}, \ldots, H^{r-1}
$$

where

$$
H^{i}=H \cdot \ldots \cdot H
$$

is the $i$-fold self-intersection of the Chern class $H$ of the bundle $\mathcal{O}(1)=\mathcal{M}_{d}$ on the scheme $\operatorname{Sym}^{d}(Y / U)$, and $r$ is the rank of the sheaf $\mathcal{F}_{d}$.

Using this, one can show that the homomorphism

$$
C H^{*}\left(\operatorname{Sym}^{d}\right) \otimes_{C H^{*}(J)} C H^{*}\left(\operatorname{Sym}^{d}\right) \rightarrow C H^{*}\left(\operatorname{Sym}^{d} \times{ }_{J} \operatorname{Sym}^{d}\right)
$$


of $C H^{*}(J)$-modules, given by the formula

$$
a \otimes b \mapsto \operatorname{pr}_{1}^{*}(a) \cdot \operatorname{pr}_{2}^{*}(b),
$$

is an epimorphism, where $\operatorname{Sym}^{d}=\operatorname{Sym}^{d}(Y / U)$ for short. In follows that the Chow group of the scheme

$$
\operatorname{Sym}^{d}(Y / U) \times{ }_{J} \operatorname{Sym}^{d}(Y / U)
$$

is generated over $C H^{*}(J)$ by the elements $\operatorname{pr}_{1}^{*}\left(H^{i}\right) \cdot \operatorname{pr}_{2}^{*}\left(H^{j}\right), i, j=0, \ldots, r-1$.

Therefore, to prove vanishing of $\left(t \times{ }_{S} t\right)_{*}\left(\pi_{1}\right)$ it suffices to show that the intersection

$$
\left(t \times{ }_{S} t\right)_{*}\left(\pi_{1}\right) \cdot \operatorname{pr}_{1}^{*}\left(H^{i}\right) \cdot \operatorname{pr}_{2}^{*}\left(H^{j}\right)
$$

is zero for any $i$ and $j$. Following [11], we will show now that, in fact, the cycle class

$$
\left(t \times{ }_{S} t\right)_{*}\left(\pi_{1}\right) \cdot \operatorname{pr}_{1}^{*}\left(H^{i}\right)
$$

vanishes for any index $i$.

Indeed, letting $\pi_{0}=\left[Z \times{ }_{U} Y\right]$, we obtain that

$$
\Delta_{Y / U} \cdot \pi_{0}=\left[Z \times{ }_{U} Z\right]
$$

and

$$
\pi_{2} \cdot \pi_{0}=\left[Z \times{ }_{U} Z\right] .
$$

Since, $\pi_{0} \cdot \pi_{0}=0$ over $U$, we also have that

$$
\pi_{1} \cdot \pi_{0}=\left(\Delta_{Y / U}-\pi_{0}-\pi_{2}\right) \cdot \pi_{0}=0 .
$$

For any natural number $n$ let $(Y / U)_{i}^{n}$ be the fibred product

$$
Y \times_{U} \cdots \times_{U} Z \times_{U} \cdots \times_{U} Y
$$

where $Z$ is located in the $i$-th place. Since $\pi_{1} \cdot \pi_{0}=0$, it follows that

$$
\left[(Y / U)_{i}^{n} \times_{U}(Y / U)^{n}\right] \cdot \pi_{1}^{(n)}=0,
$$

where $\pi_{1}^{(n)}=\pi_{1} \otimes \cdots \otimes \pi_{1}$ is the $n$-fold tensor power of the correspondence $\pi_{1}$.

Since $H$ is the class of the closed subscheme $M_{d}$ in $\operatorname{Sym}^{d}(Y / U)$,

$$
t^{*}(H)=\sum_{i=1}^{d}\left[(Y / U)_{i}^{d}\right],
$$


whence

$$
\operatorname{pr}_{1}^{*} t^{*}(H)=\sum_{i=1}^{d}\left[(Y / U)_{i}^{d} \times_{U}(Y / U)^{d}\right]
$$

Since

$$
\left[(Y / U)_{i}^{d} \times_{U}(Y / U)^{d}\right] \cdot \pi_{1}^{(d)}=0,
$$

for each index $i$, it follows that

$$
\operatorname{pr}_{1}^{*} t^{*}(H) \cdot \pi_{1}^{(d)}=0
$$

By the projection formula,

$$
\begin{aligned}
\left(t \times_{U} t\right)_{*}\left(\pi_{1}^{(d)}\right) \cdot \operatorname{pr}_{1}^{*}(H) & =\left(t \times_{U} t\right)_{*}\left(\pi_{1}^{(d)} \cdot\left(t \times_{U} t\right)^{*} \operatorname{pr}_{1}^{*}(H)\right) \\
& =\left(t \times{ }_{S} t\right)_{*}\left(\pi_{1}^{(d)} \cdot \operatorname{pr}_{1}^{*} t^{*}(H)\right)=0 .
\end{aligned}
$$

Proposition 3.3 is a local version of Theorem B. To prove it globally over $S$, we need some more preparation. Let $C$ be a rigid tensor $\mathbb{Q}$-linear category with the monoidal unity $\mathbb{1}$. For any object $M$ in $C$ one can define the trace homomorphism

$$
\operatorname{tr}_{M}: \operatorname{End}_{\mathrm{C}}(M) \rightarrow \operatorname{End}_{\mathrm{C}}(\mathbb{1})
$$

see [5, p. 113] for the details. A morphism $f: M \rightarrow N$ in C is said to be numerically trivial if, for any morphism $g: N \rightarrow M$ in $\mathrm{C}$, we have that

$$
\operatorname{tr}_{M}(g \circ f)=0
$$

Lemma 3.4 Let $M$ be a finite-dimensional object in $C$. Then any numerically trivial endomorphism of $M$ is nilpotent in the associative ring $\operatorname{End}(M)$.

Proof See [2, Proposition 9.1.14].

Now, let $\eta$ be the generic point of $S$, let $L$ be any field extension of the field $\kappa(\eta)$, and let $\xi$ be the spectrum of the field $L$. Consider the base change functor

$$
F_{\xi}: \mathrm{M}(S) \rightarrow \mathrm{M}(\xi)
$$

induced by the morphism $\xi \rightarrow S$. For any motive $M=(X, \Sigma, m)$ over $S$ the value $F_{\xi}(M)$ is the motive $M_{\xi}=\left(X_{\xi}, \Sigma_{\xi}, m\right)$ over $\xi$, where

$$
X_{\xi}=X_{\eta} \times_{\eta} \xi
$$

and $\Sigma_{\xi}$ is the pullback of the correspondence $\Sigma \in C H_{S}^{0}(X, X)$ to $C H^{0}\left(X_{\xi}, X_{\xi}\right)$, induced by the morphism from $X_{\xi} \times X_{\xi}$ to $X \times{ }_{S} X$. The functor $F_{\xi}$ can be also viewed as specialisation of Chow motives over $S$ to the point $\xi$. 
The functor $F_{\xi}$ preserves tensor products, and if $\mathbb{1}_{S}$ and $\mathbb{1}_{\xi}$ are the tensor unities in the categories $\mathrm{M}(S)$ and $\mathrm{M}(\xi)$ respectively, then

$$
F_{\xi}\left(\mathbb{1}_{S}\right)=\mathbb{1}_{\xi}
$$

Since, moreover,

$$
\operatorname{End}\left(\mathbb{1}_{S}\right)=C H_{S}^{0}(S, S)=\mathbb{Q} \quad \text { and } \quad \operatorname{End}\left(\mathbb{1}_{\xi}\right)=C H^{0}(\xi, \xi)=\mathbb{Q}
$$

the homomorphism

$$
\operatorname{End}\left(\mathbb{1}_{S}\right) \rightarrow \operatorname{End}\left(\mathbb{1}_{\xi}\right)
$$

induced by $F_{\xi}$, is an isomorphism.

Lemma 3.5 Let $M$ be a finite-dimensional object in $\mathrm{M}(S)$, and let $\Phi$ be an endomorphism of $M$ in $\mathrm{M}(S)$. Assume that the correspondence $F_{\xi}(\Phi)$ is nilpotent modulo numerical equivalence, i.e., there exists a natural number $n$ such that the correspondence $F_{\xi}(\Phi)^{n}=F_{\xi}\left(\Phi^{n}\right)$ is numerically trivial in $\mathrm{M}(\xi)$. Then $\Phi$ is nilpotent also in $\mathrm{M}(S)$.

Proof Any tensor functor between rigid categories commutes with traces, see [5, p. 116]. Therefore, for any endomorphism $g: M \rightarrow M$, we obtain that

$$
F_{\xi}\left(\operatorname{tr}\left(g \circ \Phi^{n}\right)\right)=\operatorname{tr}\left(F_{\xi}\left(g \circ \Phi^{n}\right)\right)=\operatorname{tr}\left(F_{\xi}(g) \circ F_{\xi}\left(\Phi^{n}\right)\right)=0
$$

But $F$ induces an isomorphism from $\operatorname{End}\left(\mathbb{1}_{S}\right)$ to $\operatorname{End}\left(\mathbb{1}_{\xi}\right)$, whence

$$
\operatorname{tr}\left(g \circ \Phi^{n}\right)=0
$$

It means that $\Phi^{n}$ is numerically trivial. Since $M$ is finite-dimensional, the correspondence $\Phi^{n}$, and hence $\Phi$, is nilpotent by Lemma 3.4.

Now we are ready to prove Theorem B globally over $S$. Consider the projectors

$$
\bar{\pi}_{0}=\left[\bar{Z} \times{ }_{S} X\right] \quad \text { and } \quad \bar{\pi}_{2}=\left[X \times{ }_{S} \bar{Z}\right],
$$

introduce the vertical correcting term $\theta$, and the correspondences $\tau_{0}$ and $\tau_{2}$, as shown above. Take

$$
\tilde{\pi}_{1}=\Delta_{X / S}-\tau_{0}-\bar{\pi}_{2}
$$

in order to get the splitting with

$$
M^{1}(X)=\left(X, \tilde{\pi}_{1}, 0\right)
$$

in the middle. We want to show that $\operatorname{Sym}^{d}\left(M^{1}(X)\right)=0$ provided $d>2 g-2$, where $g$ is the genus of the relative curve $X / S$. 
Consider the obvious pullback tensor functor

$$
F_{U}: \mathrm{M}(S) \rightarrow \mathrm{M}(U)
$$

sending a motive $M=(X, \Sigma, m)$ over $S$ to the motive $\left(X_{U}, \Sigma_{U}, m\right)$ over $U$, where $X_{U}=X \times{ }_{S} U$ and $\Sigma_{U}$ is the pullback of the correspondence $\Sigma \in C H_{S}^{0}(X, X)$ to the group $C H_{U}^{0}\left(X_{U}, X_{U}\right)$.

Let $\Sigma=\operatorname{Sym}^{n}\left(\tilde{\pi}_{1}\right)$ be the projector of the motive $\operatorname{Sym}^{d}\left(M^{1}(X)\right)$. Its pullback $\Sigma_{U}$ over $U$ is 0 by Proposition 3.3. Since the functor $F_{\xi}$ factorises through the functor $F_{U}$, it follows that the specialisation $\Sigma_{\xi}$ of the projector $\Sigma$ at $\xi$ is 0 too. Then $\Sigma$ is nilpotent by Lemma 3.5. Since $\Sigma$ is an idempotent, $\Sigma=0$. This finishes the proof of Theorem B.

Applying Lemma 3.5 to objects in $\mathrm{A}(S)$, which are finite-dimensional by Theorem B, we obtain Corollary C.

Acknowledgements The first version of this paper was written long ago, and I am grateful for two useful discussions - one with Ivan Panin in Princeton in 2004, and the other one with Shun-Ichi Kimura in Oberwolfach in 2006 .

Open Access This article is distributed under the terms of the Creative Commons Attribution 4.0 International License (http://creativecommons.org/licenses/by/4.0/), which permits unrestricted use, distribution, and reproduction in any medium, provided you give appropriate credit to the original author(s) and the source, provide a link to the Creative Commons license, and indicate if changes were made.

\section{References}

1. Andre, Y.: Une Introduction Aux Motifs. Panoramas et Synthèses, vol. 17. Société Mathématique de France, Paris (2004)

2. André, Y., Kahn, B.: Nilpotence, radicaux et structure monoïdales (with appendix by P. O’Sullivan). Rend. Sem. Mat. Univ. Padova 108, 107-291 (2002)

3. Bosch, S., Lütkebohmert, W., Raynaud, M.: Néron Models. Ergebnisse der Mathematik und ihrer Grenzgebiete (3), vol. 21. Springer, Berlin (1990)

4. Chow, W.-L.: The Jacobian variety of an algebraic curve. Amer. J. Math. 76(2), 453-476 (1954)

5. Deligne, P., Milne, J.S.: Tannakian categories. In: Deligne, P., et al. (eds.) Hodge Cycles, Motives, and Shimura Varieties. Lecture Notes in Mathematics, vol. 900, pp. 101-228. Springer, Berlin (1982)

6. Fulton, W.: Intersection Theory. Ergebnisse der Mathematik und ihrer Grenzgebiete (3), vol. 2. Springer, Berlin (1984)

7. Kleiman, S.L.: The Picard scheme. In: Fantechi, B. et al. (eds.) Fundamental Algebraic Geometry. Mathematical Surveys and Monographs, vol. 123. American Mathematical Society, Providence (2005)

8. Grothendieck, A.: Éléments de Géométrie Algébrique III. Étude Cohomologique des Faisceaux Cohérents. Rédigés avec la collaboration de J. Dieudonné (EGA III(1)). Publications Mathématiques IHÉS, vol. 11. Institut des Hautes Études Scientifiques, Paris (1961)

9. Hartshorne, R.: Algebraic Geometry. Graduate Texts in Mathematics, vol. 52. Springer, New York (1977)

10. Iversen, B.: Linear Determinants with Applications to the Picard Scheme of a Family of Algebraic Curves. Lecture Notes in Mathematics, vol. 174. Springer, Berlin (1970)

11. Kimura, S.-I.: Chow groups are finite dimensional, in some sense. Math. Ann. 331(1), 173-201 (2005)

12. Künnemann, K.: Arakelov Chow groups of abelian schemes, arithmetic Fourier transform, and analogues of the standard conjectures of Lefschetz type. Math. Ann. 300(3), 365-392 (1994)

13. Mattuck, A.: Symmetric products and Jacobians. Amer. J. Math. 83(1), 189-206 (1961)

14. Mattuck, A.: Picard bundles. Illinois J. Math. 5(4), 550-564 (1961)

15. Milne, J.S.: Abelian Varieties (2008). www.jmilne.org/math/CourseNotes/AV.pdf 
16. Revêtements Étales et Groupe Fondamental. Séminaire de Géométrie Algébrique du Bois Marie 1960/61 (SGA 1). Dirigé par A. Grothendieck. Augmenté de deux exposés de M. Raynaud. Lecture Notes in Mathematics, vol. 224. Springer, Berlin (1971)

17. Schwarzenberger, R.L.E.: Jacobians and symmetric products. Ill. J. Math. 7(2), 257-268 (1963)

18. The Stacks Project. https://stacks.math.columbia.edu/

19. Théorie des Intersections et Théorème de Riemann-Roch. Séminaire de Géométrie Algébrique du Bois Marie 1966/67 (SGA 6). Dirigé par P. Berthelot, A. Grothendieck et L. Illusie. Lecture Notes in Mathematics, vol. 225. Springer, Berlin (1971)

20. Théorie des Topos et Cohomologie Étale des Schémas. Tome 3. Séminaire de Géométrie Algébrique du Bois Marie 1963/64 (SGA 4). Dirigé par M. Artin, A. Grothendieck et J.L. Verdier. Lecture Notes in Mathematics, vol. 305. Springer, Berlin (1972)

21. Weil, A.: Variétés abéliennes et courbes algébriques. Publ. Inst. Math. Univ. Strasbourg 8 (1946) 